FORMATION Formation emploi

Revue française de sciences sociales

112 | octobre-décembre 2010

Pêle-mêle

\title{
Mobilité internationale et carrières des cadres : figure imposée ou pari risqué ?
}

International mobility and careers for managers: an imposed model or a risky gamble?

Internationale Mobilität und Managerlaufbahn : Pflichtübung oder Risiko?

Movilidad internacional y carreras de los gerentes : figura impuesta o apuesta arriegada?

Cécile Guillaume et Sophie Pochic

\section{(2) OpenEdition}

Journals

Édition électronique

URL : http://journals.openedition.org/formationemploi/3172

DOI : 10.4000/formationemploi.3172

ISSN : 2107-0946

Éditeur

La Documentation française

Édition imprimée

Date de publication : 30 décembre 2010

Pagination : $39-52$

ISSN : 0759-6340

Référence électronique

Cécile Guillaume et Sophie Pochic, « Mobilité internationale et carrières des cadres : figure imposée ou pari risqué ? », Formation emploi [En ligne], 112 | octobre-décembre 2010, mis en ligne le 07 janvier 2013, consulté le 30 octobre 2020. URL : http://journals.openedition.org/formationemploi/3172 ; DOI : https://doi.org/10.4000/formationemploi.3172 


\title{
Mobilité internationale et carrières des cadres : figure imposée ou pari risqué ?
}

\author{
Cécile Guillaume et Sophie Pochic*
}

\begin{abstract}
Malgré la relative appétence des cadres pour l'international, l'expérience et la rentabilité d'une mobilité internationale varient fortement suivant le contexte de départ, l'activité et le profil de la personne, mais aussi les points d'entrée dans le groupe (siège ou filiale).
\end{abstract}

L'internationalisation des marchés du travail, en lien avec la globalisation de l'économie et l'apparition de « global cities» (comme Londres, New York ou Tokyo), est présentée dans de nombreux essais sociologiques comme une tendance inéluctable modifiant en profondeur les parcours de carrières des salariés qualifiés (Sassen, 2001 ; Beck, 2001 ; Castells, 2000). L'accélération des flux de capitaux, d'informations, et désormais de compétences, au sein d'entreprises multinationales, ferait advenir une nouvelle figure de «managers transnationaux » au centre de ces flux (Doyle, Nathan, 2001). Cette migration qualifiée est parfois appréhendée sous l'angle de la « fuite des cerveaux» du Sud vers le Nord (Ennafaa, Paivandi, 2008 ; Vinokur, 2008), mais aussi en termes de transfert de savoirs et de circulation des élites ; ces mouvements étant soutenus par des politiques nationales et supranationales qui organisent une immigration «choisie » en fonction des besoins de main-d'œuvre au sein de chaque territoire (Favell, Smith, 2008).

Moins attentives à ces dimensions politiques, les rares enquêtes sociologiques sur les «cadres inter-
* Cécile Guillaume est sociologue, maître de conférences, université de Lille 1, membre du CLERSE (Centre lillois d'études et de recherches sociologiques et économiques). Elle a publié récemment : "Les enjeux de carrière dans une entreprise en restructuration permanente : la face cachée du management à France Télécom », in P. Bouffartigue, C. Gadea, S. Pochic (dir.), Cadres, classes moyennes, vers l'éclatement?, Armand Colin (sous presse, à paraître en janvier 2011); " La fabrication organisationnelle des dirigeants : un regard sur le plafond de verre», Travail, Genre et Société, n 17, 2007, pp. 79-103 (avec S. Pochic).

Sophie Pochic est sociologue, chargée de recherche, membre du CMH (Centre Maurice Halbwachs) - EHESS-ENS - École des hautes études en sciences sociales/École normale supérieure-CNRS. Elle a publié récemment: Cadres, classes moyennes, vers l'éclatement?, Armand Colin (sous presse, à paraître en janvier 201 1), ouvrage coordonné avec P. Bouffartigue et C. Gadéa. "Les carrières des cadres au coeur des restructurations: I'ambivalence des effets de genre ", Sociologie du travail, vol. 51, n² 2, 2009 , pp. 275-299 (avec C. Guillaume). 
nationaux 》 montrent comment cette expérience de la mobilité géographique s'inscrit dans des stratégies individuelles et familiales de mobilité/ reproduction sociale. L'entrée par des lycées internationaux, situés en France (Wagner, 1998), permet de décrypter les mécanismes de la socialisation des élites dirigeantes au cosmopolitisme et la formation d'un «capital international» (connaissance des langues et des cultures étrangères). Une enquête sur les expatriés résidant dans des capitales d'Europe du Nord - Londres, Amsterdam et Bruxelles (Favell, 2008) - rappelle comment la mobilité internationale peut aussi représenter une opportunité pour les jeunes diplômés, notamment femmes et issus de l'Europe du Sud, de contourner des marchés du travail discriminants ou segmentés. Ces recherches s'intéressent en revanche assez peu aux contextes de travail dans lesquels évoluent ces individus (Beaverstock, 2005). Elles tendent à mettre sur le même plan les « corporate mobility » (au sein d'une entreprise et à l'initiative des employeurs) et celles s'inscrivant dans des projets plus personnels (études, voyages, amours). Ces enquêtes évoquent certes la pluralité et l'instabilité du rapport à la mobilité selon des dimensions privées (sexe, âge, cycle de vie, nationalité), à travers notamment la question de l'éventuelle réinsertion dans le pays d'origine; cependant, elles négligent souvent les caractéristiques liées au marché du travail (secteur d'activité ou métier).

Ce modèle du « manager international » est encore peu répandu ${ }^{1}$. Pourtant, il est valorisé depuis longtemps par les multinationales et les consultants (Villette, 1991) et, depuis la fin des années 80, par les écoles de commerce puis d'ingénieurs ; celles-ci présentent en effet le stage ou l'expérience à l'étranger comme la «clé » pour faire carrière (Lazuech, 1998 ; Cerdin, 2007). Au sein des multinationales, les opportunités d'expérience professionnelle à l'étranger dépendent du degré d'internationalisation de l'activité ; rares

\footnotetext{
1 Les ingénieurs diplômés, groupe privilégié en termes de carrière au sein des cadres Français, ne sont que $13 \%$ en 2009 à travailler à l'étranger (principalement Suisse, USA, Allemagne, GrandeBretagne, Belgique) et seuls $6 \%$ ont un double diplôme obtenu à l'étranger (CNISF, 2010). La dimension internationale de leur formation a en revanche été renforcée dans les années 2000 ; ainsi, les moins de 30 ans sont $29 \%$ à avoir effectué un stage d'au moins trois mois dans une entreprise à l'étranger et $18 \%$ un stage d'au moins trois mois dans une université, alors que ces taux chutent respectivement à $14 \%$ et $8 \%$ pour les plus de 30 ans, formés dans les années 90.
}

sont encore les entreprises qui ont une gestion incitative et planifiée des mobilités transnationales (APEC, 2005). Dans cet article, nous nous proposons précisément d'étudier les formes de mobilité internationale des cadres dans une entreprise française du secteur gazier, en voie d'internationalisation. Nous montrerons, dans une première partie, comment cette offre de mobilité à l'intérieur du groupe demeure restreinte

\section{Encadré 1}

$$
\begin{gathered}
\text { Méthodologie : enquête croisée } \\
\text { sur la maison-mère et deux filiales } \\
d^{\prime} \text { 'un groupe français }
\end{gathered}
$$

L'histoire interne du processus d'internationalisation du Groupe a tout d'abord été retracée par des entretiens auprès de cadres responsables ou acteurs de cette politique de fusions-acquisitions, dans différentes directions de la maison-mère à Paris (20 cadres, dont 6 étrangers impatriés), complétés par l'étude de documents d'entreprise. Centrées au départ sur le marché interne de la maisonmère, nous avons analysé les parcours de carrière de 80 cadres de l'entreprise, dans des directions plus ou moins ouvertes à l'international, en 2005. Nous avons ensuite réalisé, en 2006, des interviews dans deux filiales européennes; I'une avec une activité de négoce d'énergie (Angleterre), l'autre de distribution de gaz (Hongrie). Ont été interviewés les cadres français expatriés là des postes de direction de la filiale, 3 hommes en Angleterre et 2 hommes en Hongrie) et les "cadres locaux " dans chaque filiale à différents niveaux de la hiérarchie 18 en Angleterre dont 7 femmes, et 18 en Hongrie dont 7 femmes). En 2008, nous sommes retournées visiter ces filiales et réaliser des entretiens complémentaires auprès de cadres et de techniciens $(10$ en Angleterre, dont 4 femmes et 12 en Hongrie, dont 5 femmes). Les entretiens en Angleterre ont été réalisés et retranscrits en anglais, sauf pour quelques salariés bilingues; la majorité des entretiens en Hongrie ont été réalisés avec un interprète, avec une traduction en consécutif et retranscrits directement en français. 
et sélective, tout en se diversifiant, en lien avec l'évolution du secteur. Pour ce faire, nous nous appuierons sur des récits de carrière auprès de cadres de la maison-mère et de ses filiales hongroises et anglaises ( $c f$. encadré 1). Nous nous centrerons ensuite sur les difficultés personnelles et les incertitudes professionnelles de cette expérience (en fonction des profils de cadres), tout en montrant la forte appétence des cadres, notamment en début de carrière, pour cette aventure internationale.

\section{ENTRE OPPORTUNITÉ PROFESSIONNELLE ET PROJET PERSONNEL : CHOISIR LA MOBILITÉ INTERNATIONALE}

Classiquement, plus l'internationalisation d'une entreprise s'accroit, plus les flux de salariés qualifiés s'intensifient et se diversifient, passant du modèle de l'expatriation (salariés du siège envoyés en filiales) à celui de l'impatriation (salariés des filiales envoyés au siège), puis pour les entreprises les plus mondialisées, à un modèle de circulation de « cadres internationaux » entre les différentes filiales (Lorrain, 2005). Bien sûr, le type de produit (service matériel ou immatériel, produit simple ou de haute technologie, matières premières) et la division internationale du travail au sein de la chaîne de production interviennent pour expliquer la trajectoire d'internationalisation et les éventuelles circulations de main-d'œuvre qualifiée. Le secteur pétrolier fonctionne ainsi, dès le départ, sur l'expatriation d'ingénieurs vers les zones d'exploration-production. Le secteur informatique quant à lui se recompose, dans les années 2000, avec la montée en puissance de l'Inde comme fournisseur d'informaticiens par exemple. Les industries de réseaux (télécommunications, eau, électricité, gaz) se sont plus récemment laissé tenter par l'international, notamment sous l'impact de l'ouverture à la concurrence du marché européen et via une stratégie d'acquisitions souvent très coûteuse. L'entreprise étudiée, spécialisée en gaz, est ainsi passée en quinze ans d'une configuration de monopole public à un groupe de taille internationale implanté dans trente pays. Elle réalise $39 \%$ de son chiffre d'affaires à l'étranger en
2006 (sur un total de 27 milliards) et évalue à $32 \%$ ses salariés hors de France en 2006 (sur un total de 50000 salariés).

Pour autant, quand on retrace les parcours de carrière des cadres en poste, l'expérience de la mobilité internationale reste faible et réservée à certains profils (en termes de niveau hiérarchique ou de métier) ${ }^{2}$. Si les autres formes de mobilité se développent progressivement (d'une filiale vers le siège, ou de filiale en filiale), les opportunités de mobilité internationale sont restreintes et les espaces de carrière restent très hiérarchisés. La figure de l'expatriation (à partir du siège) est encore ici dominante, matériellement et symboliquement.

\section{L'expatriation d'une génération à l'autre: des formes de mobilité et des profils diversifiés}

Jusqu'à récemment, l'entreprise fonctionnait sur le modèle de "l'EPIC franco-français ». L'essentiel des expériences d'expatriation était lié à l'activité d'approvisionnement en gaz en Algérie ou en Russie, avec notamment des missions d'ingénierie et d'appui technique à des entreprises étrangères partenaires. À partir de la fin des années 80, la chute du mur de Berlin a permis d'acquérir intégralement, ou via des prises de participation, les infrastructures d'anciennes entreprises publiques (la Hongrie dès 1995). Il s'agissait de sécuriser l'approvisionnement en gaz naturel, notamment avec le principal fournisseur, le russe Gazprom. Depuis 2000, l'entreprise a investi dans des licences d'exploration et de production de gaz naturel dans dix pays. Parallèlement, elle a développé des activités de commercialisation et de «trading 》 de gaz et d'électricité (achat à court et long terme), en rachetant parfois des entités déjà existantes comme en Angleterre. Enfin, pour diversifier ses activités et fidéliser ses clients, l'entreprise a développé une offre de services (gestion des déchets, nettoyage industriel,

\footnotetext{
2 Nous n'avons pas eu accès aux données statistiques du personnel en matière de mobilité internationale, mais le nombre de cadres expatriés français à l'étranger ne devait pas excéder une centaine de personnes sur un effectif global de 9000 cadres, au moment de l'enquête.

3 Établissement Public à Caractère Industriel et Commercial assurant une activité de service public (énergie, transport, télécommunications, culture), transformé en Société Anonyme en 2004 dans le cas étudié.
} 
climatisation/chauffage) via l'acquisition de filiales françaises et européennes.

Dans ce contexte, de nouvelles opportunités de carrière se sont ouvertes pour des cadres dirigeants, en charge du rachat des filiales puis de leur direction localement. En dépit du coût, le choix d'envoyer des expatriés dans les filiales rachetées est lié à trois motifs récurrents : le transfert de savoir-faire, le contrôle des activités et la volonté de susciter la confiance des partenaires locaux (banques, clients, fournisseurs) par la présence d'un "émissaire» du groupe (Beaverstock, 2005). Ces premiers expatriés, ingénieurs polytechniciens pour la plupart, n'avaient pas eu d'exposition à l'international lors de leur formation dans les années 70. Ils l'ont expérimenté lors de leur passage dans la direction des approvisionnements ("appros»). Mariés avec une femme généralement inactive et de grands enfants, ils acceptent, au début des années 2000, l'offre qui leur est faite d'aller diriger la filiale rachetée; cela leur permet de renouer avec une activité opérationnelle qu'ils ont progressivement délaissée à mesure qu'ils gravissaient les échelons hiérarchiques, et de ré-ouvrir leur espace de carrière. Il s'agit pour eux souvent d'une occasion unique, d'une durée de trois à quatre ans, qui ne se reproduira plus :

"Alors, j'ai été expatrié il y a plus de vingt ans de ça, de 80 à 82 en Algérie, dans le cadre de contrats d'assistance technique que l'entreprise avait à cette époque là avec Sonatrach. Je suis revenu à Paris ; au gré des missions de carrière, je me suis trouvé dans un business un peu nouveau puisqu'il a vraiment démarré en 97, 98 qui est celui de l'exploration - production de gaz. Donc j'ai été adjoint de cette direction jusqu'à ce qu'on achète cette boîte en Allemagne en mai 2003. On a préparé l'intégration de la filiale dans le groupe. Làj 'étais à Paris mais j'étais quand même physiquement assez souvent là-bas et puis ensuite la question s'est posée de savoir si on envoyait un patron français là-bas ou pas. Il a été décidé d'en envoyer un et mon chef m'a demandé d'y aller. Je n'ai pas trouvé la bonne raison pour lui dire non, donc j'y suis allé. » (Dirigeant homme, français travaillant au siège, 55 ans, polytechnicien, marié, femme inactive, 2 grands enfants)

Au fur et à mesure du développement de l'activité internationale de l'entreprise et de la nécessité de faire
« tourner» les expatriés en charge de la direction des filiales, des dirigeants plus jeunes se sont vu offrir la possibilité de partir. Dans le sillage des pionniers, ils ont accepté l'expatriation, voire l'ont sollicitée, notamment pour offrir un « capital international » (Wagner, 1998) à leurs enfants encore jeunes. D'où leur vigilance à l'égard des destinations proposées et des attraits symboliques (pays considérés comme «plus avancés » ou anglophones par exemple) et matériels du pays d'accueil pour leur famille. L'autre intérêt de cette opportunité de carrière est d'expérimenter une position de «dirigeant de PME », avec une autonomie de décision et d'action inégalée depuis leur passage en direction d'unité opérationnelle (transport, distribution). Pour ces hommes cadres dirigeants, généralement fort bien dotés sur le plan scolaire et déjà très hauts placés dans la hiérarchie, l'international est moins une stratégie de mobilité sociale (Favell, 2008) qu'une façon de conforter et reproduire une position dominante (Wagner, 2008), en bénéficiant notamment d'avantages matériels non négligeables; même si, dans une politique générale de réduction des coûts, les entreprises semblent plus regardantes sur les «packages ${ }^{4} »$ offerts aux expatriés :

"À Budapest, je retrouve une capacité de générer des facultés qui, au siège, me paraissent extrêmement bridées. Un intérêt intellectuel, un intérêt d'autonomie de la personnalité, un intérêt de prise de décision rapide. Quand on blaguait à table en disant qu'il $y$ a beaucoup de gens intelligents à Paris, ça veut dire que clairement, il y a une certaine neutralisation des intelligences au siège, et qu'ici, que ce soit pour des petites ou des grandes décisions, de toute façon, on est obligé de la prendre, parce qu'il n'y a pas trois directeurs au dessus. On est dans une PME. » (Dirigeant homme, français expatrié en Hongrie, 50 ans, École Supérieure de Commerce de Paris, divorcé, femme active, 3 enfants).

À côté de ces figures classiques d'expatriation « en famille » pour des postes de direction se développent également des formes de mobilité plus courtes, plus ponctuelles (et moins coûteuses pour l'entreprise), comme dans la plupart des entreprises (Morley,

\footnotetext{
${ }^{4}$ Niveau de salaire, primes et type de contrat de travail, mais aussi avantages en nature : logement, voiture de fonction, frais de scolarité des enfants, voyages, personnel domestique, éventuelle
} formation ou aide à la recherche d'emploi pour la/le conjoint-e-... 
Heraty, 2004). Ces mobilités concernent des jeunes cadres dotés de compétences expertes en financement, en droit ou dans des domaines techniques très particuliers. Les moments de rachat et les activités fortement internationalisées (finance, négoce) sont particulièrement propices à ces mobilités courtes (missions de trois à six mois, qui se prolongent parfois au coup par coup) ou faites d'allers-retours constants. Moins avantageuses sur le plan financier et contractuel, ces formes de mobilité font écho à un appétit pour l'international, développé lors de stages ou de semestres d'études dans les années $90^{5}$ (Lazuech, 1998), ou encore de formes nouvelles de coopération pour les jeunes hommes (VSNE, et plus récemment VIE ${ }^{6}$ ). Ces jeunes cadres, souvent célibataires ou récemment en couple, sont dotés de compétences linguistiques. Ils tentent l'aventure pour l'intérêt professionnel de l'expérience - notamment le développement de savoir-faire spécifiques directement liés à l'accompagnement de l'internationalisation du Groupe mais aussi pour l'intérêt personnel de l'expérience. Des travaux récents sur les mobilités à l'initiative des salariés soulignent l'importance des dimensions privées dans les projets d'expatriation et la diversification des profils, avec notamment une féminisation des élites transnationales (Favell, 2008 ; Yeoh, Willis, 2005). Certaines jeunes femmes cadres se sont forgé une solide expérience internationale via un début de carrière dans des secteurs très internationalisés (finance, droit, conseil) ou une expérience de travail à l'étranger. Elles ont justement été recrutées pour apporter cette compétence rare à l'entreprise :

"Je suis sortie en 1988, donc en 1985 j'ai fait mes deux premières années à Sup de Co Lyon, en deuxième année avec des cours d'équivalence, et je suis partie

\footnotetext{
${ }^{5}$ Particulièrement dans les écoles de commerce de rang intermédiaire, de type ESC (École Supérieure de Commerce), qui se sont distinguées dans le champ des " grandes écoles " par l'envoi des étudiants en stage à l'étranger. Les grandes écoles ont davantage adopté une stratégie de «double diplôme » avec des institutions étrangères prestigieuses, à l'exception de Polytechnique, qui a préféré augmenter son nombre d'étudiants étrangers (Lazuech, 1998).

${ }^{6}$ Le VSNE, Volontariat du Service National à l'Etranger (depuis la fin des années 70), permettait à des jeunes diplômés de se faire une première expérience " professionnelle » en entreprise à l'étranger de plus d'un an sur un statut de "volontaire » (bénévole indemnisé), tout en contournant le service militaire. Le VIE, Volontariat International en Entreprise, instauré depuis 2000, prolonge ce principe et est désormais mixte.
}

aux États-Unis faire un semestre d'été, un semestre d'automne, un semestre de printemps. J'ai eu mon $M B A^{7}$, je suis rentrée, $j^{\prime}$ 'ai eu mes examens à Lyon, et dans la foulée j'avais déjà été recrutée par Alsthom, depuis les États-Unis. Je voulais aller aux États-Unis étudier, c'était une obsession depuis le lycée, bon l'allemand est ma $1^{r e}$ langue, l'anglais la $2^{e}$, j'ai fait de l'espagnol à Sup de Co Lyon et ensuite chez Alsthom, j'ai appris l'espagnol. À titre personnel, j'adore les voyages et j'aime beaucoup l'international, ça m'a toujours attirée, ce qui fait qu'effectivement comme j'ai été approchée par Alsthom qui cherchait un profil commercial... » (Dirigeante femme, direction financière, 37 ans, célibataire, sans enfant)

\section{L'impatriation : entre stratégie de distinction et faiblesse des opportunités}

Les possibilités d'expatriation semblent se développer pour les cadres français ; à l'inverse, l'autre versant de la mobilité internationale, l'impatriation depuis les filiales étrangères, reste modeste. Il ne concerne en effet que des cadres parlant très bien le français, ce qui semble exclure les anglo-saxons, peu versés dans l'apprentissage des langues. En revanche, dans les pays de l'Est, de nombreux jeunes cadres ont précisément fait le choix de se doter de compétences linguistiques dès les années 90, notamment ceux issus de milieux cultivés. Pour ce faire, ils ont fréquenté le lycée français, suivi des filières Erasmus ${ }^{8}$ ou fait leurs études en France (système de bourses par l'Institut français), pour élargir leurs opportunités de carrière :

"Je suis née en Roumanie, peut-être que c'est plus facile pour moi le français. À l'université, j'ai commencé à étudier le français. Après, j'ai eu la possibilité de faire un mois de stage en France, et après, un semestre universitaire au sein du programme Erasmus, à Angers, à l'École Supérieure d'Agriculture. Là aussi, j'ai étudié le commerce, le marketing, et la gestion de l'environnement. " (Cadre, femme hongroise travaillant dans la filiale hongroise, 29 ans, en couple, sans enfant)

\footnotetext{
${ }^{7}$ Master of Business Administration.

${ }^{8}$ D'autres enquêtes ont montré la sélectivité sociale des programmes Erasmus (Ballatore, Bloss, 2008).
} 
Cette stratégie de distinction caractéristique de la formation des élites, en particulier en Hongrie (Gradvohl, 2006), peut également s'interpréter comme une stratégie de survie pour les cadres dirigeants locaux qui ont entrepris d'apprendre le français tout seuls, suite au rachat de leur entreprise (en sachant que la langue de travail au sein du groupe est le français). Les migrations répondent souvent à la saturation du marché du travail local. Elles doivent ici être replacées dans le contexte de l'accroissement des migrations des pays de l'Est vers l'Europe de l'Ouest, depuis la chute du mur de Berlin (Metz-Goeckel, Morokvasic-Muller et Sengatana Munst, 2007). De nombreux jeunes diplômés en provenance des pays de l'Est sont intéressés par une expérience d'impatriation en France pour les avantages financiers qui en découlent et la qualité de vie attendue (Pierre, 2003 ; Favell, 2008); de même que pour la plus-value que cela représente pour leur $\mathrm{CV}$, y compris dans la perspective d'un retour dans leur pays (compte tenu du poids croissant des multinationales dans l'économie locale).

Cela dit, les opportunités d'impatriation en France restent faibles dans le groupe étudié. Elles dépendent largement des relations avec les salariés du siège et les expatriés en filiales. Ces réseaux de travail permettent d'être informé des opportunités qui se libèrent et de court-circuiter les processus classiques de recrutement. Leur rôle est d'autant plus crucial que les filiales rachetées sont en phase de restructuration (Pochic, Guillaume, 2009). Certains hongrois, et notamment les cadres dirigeants ou les nouveaux embauchés, ont eu l'opportunité de passer quelques mois en France pour un «stage d'intégration » plus ou moins long, le plus souvent sans leur famille. Quand ils ont l'opportunité de venir au siège, c'est généralement pour des formations ou pour des réunions de " reporting ${ }^{9}$ ou de coordination par filières de métiers (Bozkurt, 2007). Les opportunités d'impatriation plus longues sont rares, en particulier pour les profils commerciaux, ceux qui ont peu de contacts avec la maison-mère ou ceux qui ont une expertise « locale » très directement liée au marché national (régulation/réglementation, par exemple).

\footnotetext{
${ }^{9}$ Rapports basés sur des indicateurs standardisés, notamment financiers, qui permettent un contrôle à distance de l'activité des filiales.
}

\section{Le « cadre international »: nouvelle figure de la mobilité au sein du groupe?}

Au-delà de ces deux figures, une troisième forme de mobilité internationale (entre filiales) émerge dans un secteur d'activité particulier et minoritaire de l'entreprise : l'exploration-production. À l'image du secteur pétrolier (Pierre, 2003), cette activité s'appuie sur une multitude de filiales, localisées dans les pays dotés en ressources énergétiques (Afrique, Russie, Mer du Nord, Moyen Orient...) ; au sein de ces filiales cohabitent des managers et experts techniques expatriés et des salariés locaux plus ou moins qualifiés. La transférabilité des compétences techniques et les besoins en main-d'œuvre qualifiée autorisent la constitution d'un marché du travail professionnel à l'échelle internationale et la circulation des élites au sein des différentes filiales d'une même entreprise ou entre entreprises ; même si les marchés internes offerts par les multinationales freinent en partie cette mobilité inter-entreprise. Les jeunes ingénieurs spécialisés en géosciences construisent donc leur carrière au sein de ce marché mondialisé, fortement structuré par d'importants acteurs opérationnels, une myriade de PME (petites et moyennes entreprises) et un marché du conseil très dynamique. Bien que le secteur soit en forte croissance, les salariés avec des profils internationaux, issus de familles mixtes et dotés de multiples compétences linguistiques, sont avantagés. Les formes de leur mobilité sont diverses (missions à l'étranger de courte durée ou expatriations/impatriations de plus longue durée) et peuvent aussi s'accompagner d'un changement d'entreprise, pour rouvrir des opportunités de promotion limitées dans des filiales de petite taille ou au contraire pour contourner la pression à l'expatriation répétée.

"Si on travaille pour BP, Shell ou Exxon, une carrière dans le métier veut normalement dire faire deux-trois ans à Aberdeen et puis partir en Afrique ou en expatriation quelque part et faire ça pendant une dizaine, une quinzaine d'années et puis commencer à viser des positions de management. Ce qui se passe dans l'industrie depuis quelques années en Angleterre, c'est qu'il y a beaucoup de petites boîtes qui se créent, et beaucoup de gens qui sont intéressés par ces petites boîtes parce qu'il y a moins de pression pour partir en 
expatriation, pour aller à droite à gauche. Beaucoup de gens ne visent qu'une carrière technique, mais d'autres visent du management et vont essayer de devenir chef de projet ou des choses comme ça dans ces petites structures. » (cadre expert, homme anglais impatrié au siège, 31 ans, ingénieur, célibataire, sans enfant)

En dehors de ce secteur d'activité, une minorité de cadres dirigeants français quadragénaires envoyés en filiale commencent à tracer la voie d'un modèle de carrière à l'international, enchaînant plusieurs postes à l'étranger, et parfois dans la même région (même si les mobilités inter-filiales restent encore faibles). Comme l'ont montré d'autres enquêtes, la possibilité d'une carrière de type « transnational » est étroitement liée au développement de compétences spécialisées accumulées au fil du temps, sur un métier ou sur une région donnés (Beaverstock, 2002 ; Wagner, 2008). Cette stratégie de carrière est pour l'heure très minoritaire. Elle est plutôt le fait de cadres moins conformes à la figure type du dirigeant français - notamment des diplômés de « petites » écoles de commerce ou universitaires (Guillaume, Pochic, 2007) qui cherchent à ouvrir leur espace de carrière en valorisant des ressources nouvelles (aptitude à travailler en contexte multiculturel, maîtrise des langues). Les ingénieurs des grandes écoles, vivier de recrutement des cadres à « haut potentiel », ont en effet pour l'instant été moins exposés à l'international, particulièrement dans le cas de passage dans les grands corps d'État. Mais cette orientation peut correspondre aussi à la poursuite d'une carrière alternative en filiales au moment où les salariés du siège sont confrontés à une lutte des places, rappelant le rôle incitatif de la saturation du marché du travail (à l'image du secteur de l'architecture design, $c f$. Kennedy, 2005 ${ }^{10}$ ). Depuis la fusion, en 2008, de l'entreprise avec un autre grand groupe européen plus internationalisé et plus décentralisé dans ses modes de fonctionnement, la raréfaction des places au siège a conduit certains dirigeants expatriés à rester à l'étranger.

\footnotetext{
${ }^{10}$ Cet auteur montre que seule une minorité d'architectes internationaux sont des cosmopolites «naturels », issus des classes supérieures. La majorité sont des cosmopolites « accidentels » qui ont été obligés de travailler à l'étranger quand le marché local était confronté à une récession, passage facilité par des réseaux créés lors de leur scolarité (Erasmus) ou par des rencontres plus fortuites.
}

En raison de son développement, notamment pour les salariés du siège, la mobilité internationale est désormais présentée comme un passage obligé pour les cadres « à potentiel », notamment dans les discours des responsables des ressources humaines. Pourtant, accepter un poste à l'étranger se révèle être un pari risqué, en raison des possibles coûts en termes de vie privée $^{11}$, mais aussi, dimension souvent négligée, en raison de sa rentabilité incertaine et variable suivant les profils de cadres et les contextes de départ.

\section{L'EXPÉRIENCE DE LA MOBILTTÉ INTERNATIONALE ET SES DIFFICULTÉS}

Qu'il s'agisse d'une expatriation proposée par la hiérarchie dans un parcours d'apprentissage de « haut potentiel » ou d'un départ risqué dans le cadre d'un projet plus personnel, ces cadres partagent une expérience professionnelle dans un contexte de travail à l'étranger marqué par des différences culturelles. Les effets de cette mobilité sur la carrière sont en outre toujours incertains au moment du départ et ne deviennent tangibles qu'au moment du retour.

\section{Stratégies familiales et résidentielles : le choix de l'installation}

Le choix de partir ou non travailler à l'étranger dépend fortement de la configuration familiale et du moment dans le cycle de vie, mais aussi de l'attachement à une région ou un pays. Dans les années 80 , cette (rare) opportunité était proposée à des hommes cadres ayant déjà atteint un certain niveau de responsabilité ou d'expertise, âgés de plus de trente ans et donc souvent déjà en couple. Le modèle traditionnel de l'expatriation de la France vers les DOM-TOM puis l'étranger était implicitement pensé en interne comme destiné à un père de famille dont la conjointe acceptait de devenir inactive, prenant en charge l'éducation des enfants pendant cette période de trois

${ }^{11}$ Dimension souvent évoquée par la littérature de sciences de gestion (Cerdin, 2007). 
ou quatre ans. À l'image de la mobilité sur le territoire national (Guillaume, Pochic, 2009) ou des carrières de diplomates (Boussard, Loriol, 2007), cette mobilité « en famille » est plus facile à négocier si la conjointe n'est pas elle-même engagée dans sa propre carrière (a fortiori si elle ne travaille pas), si elle ne pénalise pas la scolarité et le bien-être des enfants (période de la petite enfance et non période d'adolescence, afin de ne pas compromettre leur scolarité et leurs amitiés) et si les temporalités de cette expatriation ne perturbent pas trop l'équilibre familial (mission de plus d'un an, correspondant aux rythmes scolaires). Comme ces conditions ne sont pas toujours réunies, plusieurs cadres masculins considérés comme à « potentiel » par leurs hiérarchiques ont refusé des propositions d'expatriation. Il s'agissait pour eux soit de préserver leur couple (refus de la conjointe d'abandonner son emploi ou de se déraciner), soit le bien-être de leur famille (pays considérés comme risqués en termes de santé ou en termes scolaires - langue rare peu utile pour les enfants, comme le hongrois). L'arrivée des enfants et surtout les stratégies éducatives en matière de scolarité à partir du collège et surtout du lycée ont tendance à « sédentariser » les familles de classes moyennes supérieures ; celles-ci souhaitant choisir un lieu de vie qui leur ressemble (Savage, Bagnall, Longhurst, 2005).

Ce modèle a priori formaté au masculin pose davantage de problèmes pour des femmes cadres ; ces dernières étant plus souvent que leurs collègues masculins en couple avec un conjoint lui-même investi dans sa propre carrière, souvent dans une autre entreprise. En raison des préjugés des hiérarchiques et/ou des directions des ressources humaines, les femmes cadres sont moins sollicitées ou retenues pour une expatriation en raison de leur moindre mobilité supposée ou de destinations considérées comme problématiques pour des raisons culturelles ou religieuses (Forster, 1999 ; Mérignac, Roger, 2006). Quand elles arrivent à contourner ces préjugés, souvent à leur propre initiative, elles envisagent souvent le célibat géographique au départ comme une solution temporaire adéquate, notamment les trentenaires sans enfant (Mérignac, 2009). Elle s'avère risquée au niveau privée si elle se pérennise, à la fois pour le couple mais aussi pour les projets de maternité (projets reportés puis abandonnés, sans véritable choix). La solution du départ en famille avec suivi du conjoint s'avère être très rare au féminin, plutôt dans les couples d'experts en milieu de carrière. Elle pose en effet des problèmes professionnels pour le conjoint, particulièrement sur certaines destinations rurales ou peu développées, comme les zones d'exploration et de production de gaz $^{12}$.

De manière évidente, la mobilité internationale est plus facile à organiser du côté des salariés en début de carrière, surtout quand elle correspond à leurs souhaits ou projets de vie. Mais dans l'entreprise étudiée ici, les missions longues sont paradoxalement proposées à des seniors déjà installés dans la vie en raison du niveau de poste concerné (des salariés qualifiés étant déjà présents dans toutes les filiales rachetées). Des jeunes experts sont plutôt sollicités pour des missions courtes, qui parfois se répètent et s'enchaînent sur une zone géographique, ce qui ne facilite pas l'installation dans le pays d'accueil. Pourtant, les échanges d'étudiants dans le cadre d'Erasmus ou de coopération sont susceptibles de créer des couples mixtes en début de carrière, avec des aspirations à la mobilité pour des raisons privées qui sont plus ou moins en cohérence avec les offres sur ce marché du travail interne du groupe multinational. Contrairement aux images stéréotypées sur la faible aspiration à la mobilité des femmes, certaines jeunes femmes diplômées, notamment universitaires, sont très attirées par des missions à l'international ; qu'il s'agisse pour elles de se distinguer en interne ou prouver aux autres ou à soi-même leur émancipation (Favell, 2008). À l'inverse, les jeunes hommes célibataires sont loin d'être tous des « nomades ». Ils peuvent ne pas en avoir l'appétence, que ce soit pour des raisons d'intégration sociale (vouloir conserver ses amis, être attaché à sa région), des raisons matérielles (avoir déjà des opportunités de promotion au niveau local, avoir acheté un appartement avec un crédit élevé et ne pas vouloir/pouvoir le louer) ou des raisons linguistiques (ne pas s'estimer capable de travailler dans une langue étrangère). Les contextes nationaux sont ici cruciaux, notamment en matière de marché immobilier; celui-ci s'avérant peu dynamique en Hongrie et extrêmement onéreux en Angleterre. En France, les cadres dirigeants

\footnotetext{
${ }_{12}$ En raison de sa récente internationalisation, le groupe n'a pas encore systématisé l'aide à la recherche d'emploi du conjoint, qui est proposée au cas par cas, en fonction du profil du cadre et de la direction. D'autres entreprises multinationales (Air Liquide, Areva, Danone, Thales, Schlumberger, entre autres) se sont associées en 2000 pour mettre en place un " pool d'échanges » d'offres d'emploi à l'international pour faciliter la mobilité des couples à double carrière (Partnerjob.com).
} 
français qui ont souvent déménagé sur le territoire national et qui ont été hébergés comme locataires dans le parc immobilier de l'entreprise ${ }^{13}$, sont parfois paradoxalement plus « mobiles » que les jeunes cadres qui ont déjà acquis un logement, notamment en région parisienne.

\section{S'intégrer à l'étranger, travailler avec les autres}

Travailler à l'étranger est loin d'être simple. Une période d'adaptation linguistique est souvent nécessaire avant de pouvoir être à l'aise dans les relations de travail et de mettre en avant ses compétences techniques et surtout relationnelles dans le cas de fonction d'encadrement ou d'animation d'équipe. L'entreprise accompagne en général la prise de poste par des cours de langues, rarement en amont (les expatriations sont rarement programmées à l'avance), mais plus souvent à l'arrivée. Ces expatriés ou impatriés prennent un poste et doivent acquérir des compétences linguistiques « sur le tas »; à part en Hongrie, où la complexité de la langue et le bilinguisme de nombreuses assistantes autorisent les expatriés à travailler en français, parfois accompagnés d'un traducteur professionnel. Ils doivent également «se débrouiller » avec de nombreux petits tracas de la vie quotidienne liés à l'installation dans un pays étranger (problèmes administratifs par exemple), alors qu'ils disposent d'assez peu de temps en dehors de leur travail. Dans cette enquête, le périmètre européen de la mobilité et sa relative organisation par l'entreprise neutralisent toutefois les difficultés classiquement liées aux politiques d'immigration (obtention de visas de séjour, clôture de marchés professionnels, autorisation de travail du conjoint, scolarisation des enfants).

Mais au-delà de la barrière de la langue, les expatriés expérimentent en situation des différences d'habitudes, de cultures, de législations, de manière de procéder, qui rendent problématique l'exercice du pouvoir et du management. L'asymétrie de pouvoir entre l'expatrié arrivant du siège vers la filiale ou l'impatrié aspiré par le siège, dans des logiques de contrôle

${ }^{13}$ Ce parc immobilier de location destiné aux agents de l'entreprise s'amenuise, dans une optique de réduction des coûts ; certains biens étant vendus à d'autres sociétés, d'autres étant cédés pour favoriser l'accès à la propriété des salariés. et/ou de transfert de compétences, prend alors tout son sens (Pierre, 2003). Prendre un poste de management, a fortiori dans une filiale en restructuration, dans laquelle la mission de l'expatrié consiste entre autres à réduire les effectifs et à réorganiser, crée des relations de travail assez tendues et de défiance avec l'encadrement local. Les horaires de travail dans les premiers mois du poste sont en général très intenses. Les cadres locaux reprochent souvent aux expatriés de mettre du temps avant de comprendre les spécificités de la filiale, tout en souhaitant imprimer leur marque assez rapidement afin d'avoir une évaluation positive par le siège de leur passage en filiale. La méconnaissance réciproque des systèmes éducatifs ou des entreprises étrangères donne souvent l'impression de part et d'autre que les compétences ne sont pas reconnues à leur juste valeur (en raison de l'absence d'équivalent des grandes écoles françaises en Europe). Prendre un poste d'expert, notamment dans des domaines techniques, génère moins de tensions dans des relations de travail plus « équilibrées », avec parfois des échanges dans les deux sens, même si le contenu du travail peut s'avérer décevant (Boussard, Loriol, 2007) :

«Paris pense toujours qu'il faut envoyer des gens de Paris dans les filiales. Coacher les filiales, c'est leur but. Mais ce n'est pas un vrai échange. C'est un contrôle. Ils utilisent l'expatriation comme un moyen de contrôler les filiales. Un peu en dessous, ils pourraient trouver les gens pour faire correctement leur travail. Et après avoir eu une expatriation à Paris, après avoir passé trois ans, faire tourner les gens dans les différents pays où il y a des filiales. Ça serait bien. Pas toujours les Français qui doivent circuler. On peut envoyer quelques Hongrois en Slovaquie, quelques Slovaques en Roumanie » (Cadre dirigeant, homme hongrois travaillant dans la filiale hongroise, 55 ans, ingénieur, marié, femme active, 2 enfants)

\section{Le poste $d^{\prime}$ après : promotion ou stagnation}

Quel que soit leur point de départ, les cadres partent avec l'idée que cette expatriation courte ou longue doit être rentable pour leur carrière ultérieure. Pourtant, à l'image des migrantes qualifiées des pays de l'Est pro-européennes qui découvrent la relative clôture et saturation du travail des professionnels au Danemark 
(Liversage, 2009) ou des informaticiens indiens aux États-Unis qui se retrouvent sur des emplois temporaires moins payés que les américains (Chakravartty, 2008), les migrants qualifiés au sein de cette grande entreprise multinationale découvrent parfois tardivement que l'entreprise ne reconnaît pas obligatoirement leurs diplômes et leur expérience antérieure, et surtout ne sait pas toujours utiliser ensuite les compétences acquises à l'international.

La rentabilité de la "corporate mobility» dépend d'abord du degré d'internationalisation de l'entreprise et de ses différentes activités. Dans le cas étudié, il faut ainsi distinguer les petites directions très ouvertes à l'international (métiers de l'exploration-production, du négoce d'énergie) qui sont contraintes de gérer une main-d'œuvre à l'échelle internationale et les directions très ancrées localement, au sein desquelles les compétences internationales ne sont ni nécessaires ni reconnues (distribution vers la clientèle ou gestion des infrastructures). Les meilleures carrières internationales sont en effet souvent celles qui permettent d'utiliser des savoir-faire nationaux : langue, réseaux d'affaires, habitudes professionnelles, normes juridiques (Wagner, 2008 ; Kennedy, 2005). En outre, au niveau du groupe, la politique de gestion de la mobilité internationale est encore très récente. Elle commence à se structurer pour la gestion des flux siège-filiales ; cependant, les différentes filiales du groupe dans un même pays, qui dépendent de directions différentes, ne se concertent pas pour offrir des opportunités de carrière.

Moins que les résultats durant la mission, ce sont surtout le contexte de départ et le type de carrière qui s'avèrent cruciaux pour comprendre les conditions de retour en termes de salaire et de promotion (ou non) à la suite de cette expérience. Dans le cas d'une expatriation à l'initiative de l'entreprise d'un « cadre à potentiel » (ou déjà dirigeant) sur un poste de direction de filiale, cette expérience est généralement suivie d'une promotion, souvent d'ailleurs sans lien avec l'international ; cela peut d'ailleurs être décevant pour les cadres qui rechignent à aller s'enfermer dans un poste de management en province. Dans un contexte de réorganisations permanentes, les retours d'expatriation sont en effet risqués ; leur poste, voire leur service ou leur direction ont pu être supprimés ou profondément transformés, avec une recomposi- tion des réseaux relationnels. Attentifs à leur « visibilité », même à $2000 \mathrm{~km}$ du siège, certains seniors très diplômés et plus expérimentés essayent d'entretenir à distance ces relations informelles. Il s'agit pour eux de se tenir au courant de l'évolution de la stratégie de la maison-mère ou du siège, et donc des opportunités de poste à venir, surtout en fin de mission.

En revanche, le retour d'expatriation des cadres partis pour occuper un poste vacant ou s'occuper d'une mission (parfois à leur initiative) sur un poste de cadre intermédiaire ou d'expert n'est souvent pas anticipé par leur hiérarchie directe (qui souvent a elle-même changé pendant la période). Bon nombre de cadres experts se retrouvent donc « oubliés» en filiale, surtout s'ils négligent d'entretenir leurs réseaux. À leur retour, ils sont considérés comme en «surnombre», en recherche interne de poste ${ }^{14}$. Ils sont parfois obligés d'accepter une stagnation de salaire, voire un déclassement en termes de responsabilités pour retrouver un emploi. Surtout, les jeunes cadres embauchés pour leurs compétences spécifiques en matière de fusion-acquisition (sur le plan juridique, financier, commercial ou communication) peinent à faire valoir un " métier» transférable ailleurs. Ils ont du mal à trouver des points de chute dans les directions opérationnelles en France, pourtant point de passage obligé pour une carrière de dirigeant; et ce d'autant plus qu'ils ont parfois bénéficié d'un avancement en grade plus rapide que les autres, ce qui les rend moins « employables » compte-tenu du lien entre niveau hiérarchique et âge dans d'autres directions plus opérationnelles :

"Soit la personne qui était partie en expatriation était dans une courbe ascendante, et de toute façon, qu'elle parte ou qu'elle ne parte pas, on savait parfaitement où elle allait aller. Sinon les autres, on les fait partir parce qu'il y a un besoin. Il part deux ou trois ans, mais au moment du retour, rien n'a été prévu: "Ben, qu'est-ce qu'on va faire de lui ?" S'il rentre, on le met dans un placard le temps qu'on lui trouve un poste plus ou moins bon, souvent pas très bon, mais de toute façon, on le prend. Soit il reste, mais il ne

\footnotetext{
${ }^{14}$ Car cette ancienne entreprise publique ne licencie pas ses salariés embauchés au "statut » national du personnel des IEG, Industries Électriques et Gazières (créé en 1946 et normalement restreint aux salariés de nationalité française et âgés de moins de 45 ans), alors qu'elle peut le faire pour ceux embauchés en CDI.
} 
faut pas rester trop longtemps à l'étranger, parce que vous êtes incasable après » (cadre femme française au siège, ayant vécu deux ans de missions au Mexique et Argentine, 33 ans, maîtrise droit Dauphine, mariée à un américain, un enfant).

Les cas d'impatriation au siège s'avèrent également souvent décevants pour les cadres des filiales; en effet, leur mission débouche rarement sur des postes d'encadrement, en raison notamment de leur manque de réseaux au siège. Leurs conditions de vie ne sont parfois pas à la hauteur de leurs espérances. C'est le cas notamment des profils d'ingénieurs sur des missions techniques (ici hongrois et allemands de l'Est) qui n'ont pas réussi à négocier une mission assez longue pour envisager de partir en famille, ou un salaire suffisant pour assumer les frais occasionnés par leurs déplacements (notamment en cas de célibat géographique). À l'inverse, en raison de la forte valorisation des profils de financiers et commerciaux des cadres de la filiale anglaise (activité de négoce d'énergie), pays considéré comme « en avance » sur la libéralisation du marché de l'énergie, des anglais peuvent davantage négocier leur salaire d'embauche ; même s'ils se plaignent d'une moindre progression de salaire par rapport à leurs collègues restés en filiale. Plusieurs anglais ou hongrois insatisfaits des conditions qui leur étaient faites ont ainsi interrompu leur mission pour revenir en filiale; ce retour précipité a été plus ou moins bien géré par la filiale (leur poste ayant parfois été octroyé à d'autres pour rouvrir des possibilités de promotion interne dans la filiale).

Ces exemples d'impatriation « ratée » jouent un rôle crucial dans les réticences des salariés des filiales à accepter une proposition ultérieure ou à se projeter dans la perspective d'une carrière de « cadre international » circulant dans les filiales du groupe. Quelques jeunes cadres étrangers ont d'ailleurs décliné l'offre qui leur était faite de travailler pour le groupe. Ils craignaient de se laisser enfermer dans des postes trop spécialisés, souvent dimensionnés à l'échelle du groupe et comportant de nombreux déplacements (par exemple, responsable de l'implantation de modules du progiciel SAP dans les filiales rachetées). Ceux qui ont réussi à obtenir une impatriation à leur initiative, pour suivre leur conjoint, pour échapper au plafonnement professionnel dans leur filiale, ou pour retrouver un emploi en cas de fermeture/revente de la filiale (cas en Allemagne ${ }^{15}$ ), ont souvent dû accepter un déclassement professionnel, en termes de niveau de poste et de salaire, comme dans les cas de mobilité de suivi du conjoint au niveau national (Pochic, Guillaume, 2009) :

"Quand on a proposé à mon mari cette opportunité, on n'avait pas trop le choix, car son poste de chef de projet pour la Libye arrivait à terme et ma mission aussi était finie, et on avait déjà tous les deux la chance d'avoir échappé aux restructurations à Berlin. Mais moi, personnellement, c'était un peu un rêve d'enfance de venir en France, j'adore la littérature française, $j$ 'avais pris russe en premier langue, mais français en deuxième langue pour pouvoir lire les cuvres en version originale. En Allemagne de l'Est, on ne pensait jamais qu'on apprenait une langue pour aller travailler à l'étranger! (...) Mais mon mari est redescendu en responsabilités, comme moi, en venant $i c i$, et aussi en termes de salaires. Même si on a une aide pour la scolarité des enfants, et une aide au logement, ça ne dure pas éternellement. On le savait avant, mais on a accepté en se disant qu'on allait pouvoir renégocier après. » (Cadre expert, femme allemande impatriée au siège, 42 ans, diplôme d'ingénieur géologue obtenu à Moscou, quadrilingue, mariée, 3 enfants, dont un fils de 18 ans resté à Berlin)

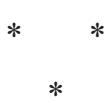

Par comparaison avec d'autres enquêtes sur des secteurs plus internationalisés comme le conseil, la finance (Beaverstock, 2005) ou l'architecture design (Kennedy, 2005), l'émergence d'une «élite transnationale » dont la particularité serait d'avoir connu une succession de postes à l'international reste très hypothétique dans l'entreprise du secteur énergétique étudiée ici, sauf peut-être sur des métiers très spécifiques (géosciences). La récente ouverture à l'international explique en partie cette situation. En effet, depuis sa fusion avec une autre entreprise plus internationalisée, en 2008, les recrutements

15 Après son rachat, l'ancienne entreprise publique d'Allemagne de l'Est est passée de 13000 salariés en 1989 à 200 en 1998, après la revente de plusieurs pans d'activité et plusieurs restructurations. Une petite minorité d'ingénieurs a réussi à conserver son poste en échange d'une expatriation sur Paris. 
« internationaux » (diplômés du supérieur avec une formation ou une expérience à l'étranger) sont privilégiés au nom d'une politique de «diversité $»^{16}$. Le type de produit et la division du travail tout au long de la chaîne de valeur interviennent aussi. Les activités traditionnelles de cette entreprise de réseaux dans le secteur énergétique (stockage, transport, distribution et commercialisation) génèrent la majorité des postes d'encadrement; or, ces activités sont très localisées (et non délocalisables) et dépendantes des contextes nationaux.

Surtout, valoriser l'international au niveau du recrutement ou en début de carrière ne signifie pas automatiquement diversifier le profil des dirigeants (tout comme la féminisation des cadres peut coexister avec le maintien du «plafond de verre »). La politique de gestion des « cadres à potentiel », qui vise à internationaliser les futurs cadres dirigeants ${ }^{17}$, demeure focalisée sur un « vivier » très franco-français, les jeunes diplômés des grandes écoles ; même si elle intègre désormais quelques cadres étrangers repérés dans les postes à responsabilités des filiales. Pour tous les autres cadres, le passage par l'international répond à des besoins ponctuels de l'entreprise ; besoins qui peuvent s'accroitre avec sa financiarisation et sa fusion avec un autre groupe internationa $1^{18}$. Cependant, ce passage reste très risqué professionnellement, peu organisé et surtout peu valorisé en interne (ce constat se retrouve

\footnotetext{
${ }^{16}$ qui met aussi l'accent sur la diversité des origines (avec le développement d'actions de recrutement dans les quartiers populaires), l'intégration des personnes handicapées, la mixité et l'égalité professionnelle hommes-femmes, et la mixité en termes d'âges.

17 à travers un passage en filiale, mais aussi des formations internationales type MBA ou l'encadrement d'équipes multiculturelles. ${ }^{18}$ Transfert de normes de gestion ou de pratiques, centralisation de certaines fonctions support, à l'image du secteur agro-alimentaire, (Seignour, Palpacuer, 2005).
}

dans de nombreux autres secteurs, $c f$. APEC, 2006). On assiste certes à l'émergence de formes de mobilité nouvelles, davantage liées à l'international et beaucoup moins à la mobilité nationale jusqu'alors privilégiée. Elles offrent des opportunités de carrière accélérée pour de jeunes cadres n'ayant pas forcément les atouts « classiques » de départ. Néanmoins, l'entreprise peine à reconnaître ces expériences au regard d'autres parcours de carrière plus classiques (diplôme d'ingénieur puis management opérationnel).

La cooptation par les pairs et les responsables hiérarchiques demeure un mécanisme central de gestion des postes et des opportunités de carrière, au siège comme dans les différentes filiales, même si le facteur «chance» joue également. Le réseau de travail constitue à la fois le gage d'une opportunité de départ, notamment sous la forme la plus valorisée de mobilité (l'expatriation), mais aussi et surtout d'un retour réussi. À l'image des mobilités des étudiants étrangers, il est nécessaire de s'interroger sur les conditions de retour (ou de non-retour). Il s'agit de réfléchir à la rentabilité objective de la mobilité internationale sur la carrière professionnelle (Ennafaa, Paivandi, 2008 ; Schomburg, Teichler, 2008). En dehors des bénéfices personnels que les salariés peuvent retirer d'une expérience à l'international, pour eux-mêmes ou pour leurs enfants, les coûts de la mobilité s'avèrent souvent dissuasifs. Le développement des mobilités ponctuelles et fréquentes (voyages d'affaires), plutôt en début de carrière, est sans doute révélateur à la fois d'une volonté de réduction des coûts, en particulier en période de crise économique, mais aussi peut-être d'une offre de mobilité plus facile à organiser ; même si cette dernière s'avère très usante dans la durée et difficile à gérer pour les couples à double carrière, et notamment pour les femmes. 


\section{Bibliographie}

APEC (2006), « La gestion des cadres dans les entreprises internationales. Comparaison des politiques et des méthodes de gestion des grandes entreprises », Les Études de l'emploi cadre, novembre.

Ballatore M., Bloss T., (2008), «L'autre réalité du programme Erasmus : affinité sélective entre établissements et reproduction sociale des étudiants ", Formation Emploi, n ${ }^{\circ} 103$, pp. 57-74.

Beaverstock J.V. (2005), "Transnational Elites in the City: British Highly-Skilled Inter-Company Transferees in New York' City's Financial District", Journal of Ethnic and Migrations Studies, 31 (2), pp. 245-268.

Beaverstock J.V. (2002), "Transnational elites in global cities: British expatriates in Singapore's financial district”, Geoforum, 33, pp. 525-538.

Beck U. (2001), La société du risque. Sur la voie d'une autre modernité, Paris, Éditions Aubier.

Bozkurt O. (2007), "Wired for work”, Society, 44 (2), pp. 42-52.

Boussard V., Loriol M. (2007), Choisir ou subir la mobilité? Stratégies de carrières et arbitrages personnels aux Affaires étrangères? Communication aux Journées Internationales de Sociologie, Londres.

Castells M. (2000), The rise of the Network Society? Oxford : Blackwell.

Cerdin J.-L. (2007), S'expatrier en toute connaissance de cause, Paris, Eyrolles.

Chakravartty P. (2008), "Symbolic Analyst or Indentured Servants? Indian High-Tech Migrants in America's Information Economy", In Favell, A., Smith M.P. (Eds), The Human Face of Global Mobility, Comparative Urban and Community Research, volume 8, pp. 159-180.

CNISF (2010), Observatoire de l'emploi des ingénieurs diplômés, Rapport du CEFI, juin.
Doyle J., Nathan M. (2001), Wherever Next? Work in a Mobile World. London : The Industrial Society.

Ennafaa R., Paivandi S. (2008), « Le non-retour des étudiants étrangers: au-delà de la fuite des cerveaux », Formation Emploi, n ${ }^{\circ} 103$, pp. 23-39.

Favell A. (2008), Eurostars and eurocities. Free movement and mobility in an integrating Europe, London: Blackwell Publishing.

Favell A., Smith M.P. (Eds) (2008), "The Human Face of Global Mobility", Comparative Urban and Community Research, vol. 8.

Forster N. (1999), "Another glass ceiling? The experience of women professionals and managers on international assignments", Gender, Work and Organization, 6 (2), pp. 79-90.

Gradvohl P. (2006), «La formation des élites hongroises et l'Occident, 1980-2000 », In Bauquet, N. Bocholier F. (Eds), Le communisme et les élites en Europe Centrale, Paris, PUF, pp. 305-322.

Guillaume C., Pochic S. (2009), "What would you accept to sacrifice? Access to top management and the work/life balance", Gender, Work and Organization, 16 (1), pp 14-36.

Guillaume C., Pochic, S. (2007), «La fabrication organisationnelle des dirigeants: un regard sur le plafond de verre », Travail, Genre et Sociétés, 17, pp. 79-104.

Kennedy J. (2005), "Joining, constructing and benefiting from the global workplace: transnational professionals in building-design industry", The Sociological Review, 53 (1), pp. 172-197.

Lazuech G. (1998), « Le processus d'internationalisation des grandes écoles », Actes de la Recherche en Sciences Sociales, 121-122, pp. 66-76.

Liversage A. (2009), "Vital conjonctures, shifting horizons: high-skilled female immigrants looking for work", Work, Employment and Society, 23 (1), pp. 120-141. 
Lorrain D. (2005), « La firme locale-globale: Lyonnaise des Eaux (1980-2004)», Sociologie du Travail, 47, pp. 340-361.

Mérignac O. (2009), «Les femmes dans le processus d'expatriation », Travail, Genre et Société, 21, pp. 131-151.

Mérignac O., Roger A. (2006), « Mobilité internationale : le cas de l'expatriation en célibataire géographique », Revue Sciences de Gestion, 52, pp. 63-79.

Metz-Goeckel S., Morokvasis-Muller M., Sengatana Munst A. (2007), Migration and Mobility in Enlarged Europe: A Gender Perspective, Verlag Barbara Burich.

Morley M., Heraty N. (2004), "International assignments and global careers. Thunderbird International Business Review”, 46 (6), pp. 633-646.

Pierre P. (2003), Mobilité internationale et identités des cadres. Des usages de l'ethnicité dans l'entreprise mondialisée, Paris, Sides.

Pochic S., Guillaume C. (2009), « Les carrières des cadres au cœur des restructurations : la recomposition des effets de genre ? », Sociologie du travail, 51 (2).

Sassen S. (2001), "Cracked cases: notes towards an analytics for studying transnational processes",
In Pries L. (Ed.), New transnational social spaces. London : Routledge.

Savage M., Bagnall G., Longhurst B. (2005), Globalization and belonging, London : Sage.

Seignour A., Palpacuer F. (2005), «Globalisation et emploi. Les nouvelles stratégies organisationnelles des multinationales agroalimentaires en Europe », Gérer et comprendre, 82, pp. 23-37.

Schomburg H., Teichler U. (2008), « Mobilité internationale des étudiants et débuts de vie active», Formation Emploi, $\mathrm{n}^{\circ}$ 103, pp. 41-55.

Villette M. (1991), «Intégrer les multinationales françaises », Gérer et comprendre, juin, pp. 16-27

Vinokur A. (2008), « De la mobilité des cerveaux », Formation Emploi, n ${ }^{\circ} 103$.

Wagner A-C. (2008), Les classes sociales dans la mondialisation, Paris, collection « Repères ».

Wagner A-C. (1998), Les nouvelles élites de la mondialisation. Une immigration dorée en France. Paris, PUF.

Yeoh B., Willis K. (2005), "Singaporean in China: transnational women elites and the negotiation of gendered identities", Geoforum, 36, pp. 211-222.

Résumé

\section{Mobilité internationale et carrières des cadres : figure imposée ou pari risqué ? \\ Cécile Guillaume et Sophie Pochic}

La mobilité internationale devient-elle un passage obligé pour les cadres? Des récits de carrières avec des salariés au siège et dans les filiales hongroise et anglaise d'une grande entreprise française du secteur énergétique permettent de saisir le développement à la fois sélectif et restreint des opportunités d'expatriation/impatriation pour les cadres et surtout les dirigeants. Malgré la relative appétence des cadres pour cette aventure, les rétributions associées à ces mouvements sont très variables suivant le profil des individus (âge, sexe, diplôme), le type d'activités exercées et surtout les points d'entrée dans le groupe (siège ou filiale).

Mots clés

Mobilité géographique, mobilité professionnelle, cadre, cheminement professionnel, économie mondiale, travailleur migrant

Journal of Economic Literature: J 61, J 24, M 51 